\section{HLA-B*40:01 Is Associated with Ankylosing Spondylitis in HLA-B27-positive Populations}

\section{To the Editor:}

Ankylosing spondylitis (AS) is a chronic inflammatory rheumatic disease that primarily involves the axial skeleton and the sacroiliac joints. The susceptibility to AS is almost entirely attributable to genetic factors; their heritability is estimated at around $90 \%{ }^{1}$. The MHC class I is the most significant genetic region for the disease, and most of its genetic association is driven by HLA-B $27^{2}$. There is evidence suggesting that the presence of other HLA-B alleles, including $B * 14, B * 38, B * 39, B * 40$, and $B * 52$, may also confer greater susceptibility to $\mathrm{AS}^{3}$. A recent study examined HLA loci associations of AS genotyping of 7264 MHC single-nucleotide polymorphisms (SNP), in 9069 affected individuals and 13,578 controls ${ }^{4}$. Genotyping data were followed by imputation of SNP, classical HLA alleles, and residues at polymorphic amino acid positions of HLA proteins. In addition to HLA-B*27, the association was identified with $B * 13: 02$, $B * 40: 01, B * 40: 02, B * 47: 01$, and HLA-B*51:01 (risk alleles); and B*07:02 and $\mathrm{B} * 57: 01$ (protective alleles).

We examined the distribution of HLA-B alleles in patients with AS and control subjects in a Spanish population previously described by our group $^{5,6}$. A total of 1300 individuals were studied as 3 distinct cohorts. Cohort 1 comprised 462 healthy controls who were HLA-B27-negative; cohort 2 included 367 patients with AS; and cohort 3 consisted of 549 controls who were HLA-B27-positive. The patients with AS were diagnosed following New York criteria ${ }^{7}$. All individuals gave written informed consent prior to enrolling in the study. The protocol was approved by the ethics committee of the Hospital Universitario Central de Asturias, Spain, and conducted according to the Declaration of Helsinki.

Table 1 shows the distribution of HLA-B alleles in controls ( $\mathrm{B} * 27$-negative and $\mathrm{B} * 27$-positive) and patients with AS $(\mathrm{B} * 27$-negative and $\mathrm{B} * 27$-positive). We have confirmed the well-established association of AS with HLA-B27, occurring in $86.7 \%$ of AS cases. No significant difference was observed between the frequencies of $B * 27$ subtypes in HLA-B27-positive populations (AS patients and controls). When we compared all patients with AS to controls who were $\mathrm{B} * 27$-negative, we observed results similar to those found in Cortes, et $a l^{4}$. B*07:02 and $\mathrm{B} * 57: 01$ allele frequencies were significantly decreased in patients with AS compared to healthy controls $\left(9.5 \%\right.$ vs $18.8 \%, \mathrm{P}_{\mathrm{BONF}}<0.01$, OR $0.45,95 \%$ CI $0.30-0.70 ; 2.7 \%$ vs $7.8 \%, 2$-tailed Fisher's exact test $\mathrm{p}<0.005$, OR 0.33 , $95 \%$ CI $0.16-0.68$; respectively). In addition, carriage of $B * 44$ and $B * 51: 01$ was also significantly decreased in patients with AS compared to controls (14.4\% vs $30.1 \%, \mathrm{P}_{\mathrm{BONF}}<10^{-5}$, OR $0.39,95 \%$ CI $0.28-0.56 ; 8.7 \%$ vs $16.9 \%, \mathrm{P}_{\mathrm{BONF}}<0.05$, OR $0.47,95 \%$ CI $0.30-0.73$; respectively).

When we analyzed only B27-positive patients with AS and B27-positive control subjects, all these differences disappeared. However, the HLA-B*40:01 allele was significantly increased in $B * 27$-positive patients with AS compared with $\mathrm{B} * 27$-positive controls $\left(\mathrm{P}_{\mathrm{BONF}}<0.001\right.$, OR 23.36, 95\% CI 3.04-179.42). In addition, $B * 38: 01$ allele frequency was significantly increased in patients with AS who were B27-negative compared to B27-negative controls $\left(20.4 \%\right.$ vs $4.6 \%, \mathrm{P}_{\mathrm{BONF}}<0.01$, OR 5.38, $95 \% \mathrm{CI}$ 2.37-12.24)

Previous findings identified a strong association between ERAP1 and AS that is restricted to HLA-B ${ }^{* 27-}$-positive disease ${ }^{8}$. The study conducted by Cortes, et al showed that HLA-B*40:01 interacts with ERAP1 in the same manner ${ }^{4}$. Several studies have suggested that HLA-B*40:01 is another HLA-B allele operative in AS susceptibility, in US and UK patients with $\mathrm{AS}^{3}$. Wei, et al reported that $\mathrm{B} * 40: 01$ is a risk factor for HLA-B27-negative AS in Taiwan Chinese ${ }^{9}$, suggesting that the effect of this allele could be different in non-white patients. In our study, HLA-B*40:01 association with AS is confirmed in HLA-B27 white populations. All these data provided evidence that HLA-B operates in AS through a mechanism involving aberrant processing of antigenic peptides. Moreover, the association across ethnic groups suggests that the association is directly with HLA-B*40:01, rather than any other gene in linkage disequilibrium with it, although further studies will be required to confirm this.

Table 1. Phenotype frequencies in HLA-B antigens in patients with AS and control groups, stratified by HLA-B27 positivity.

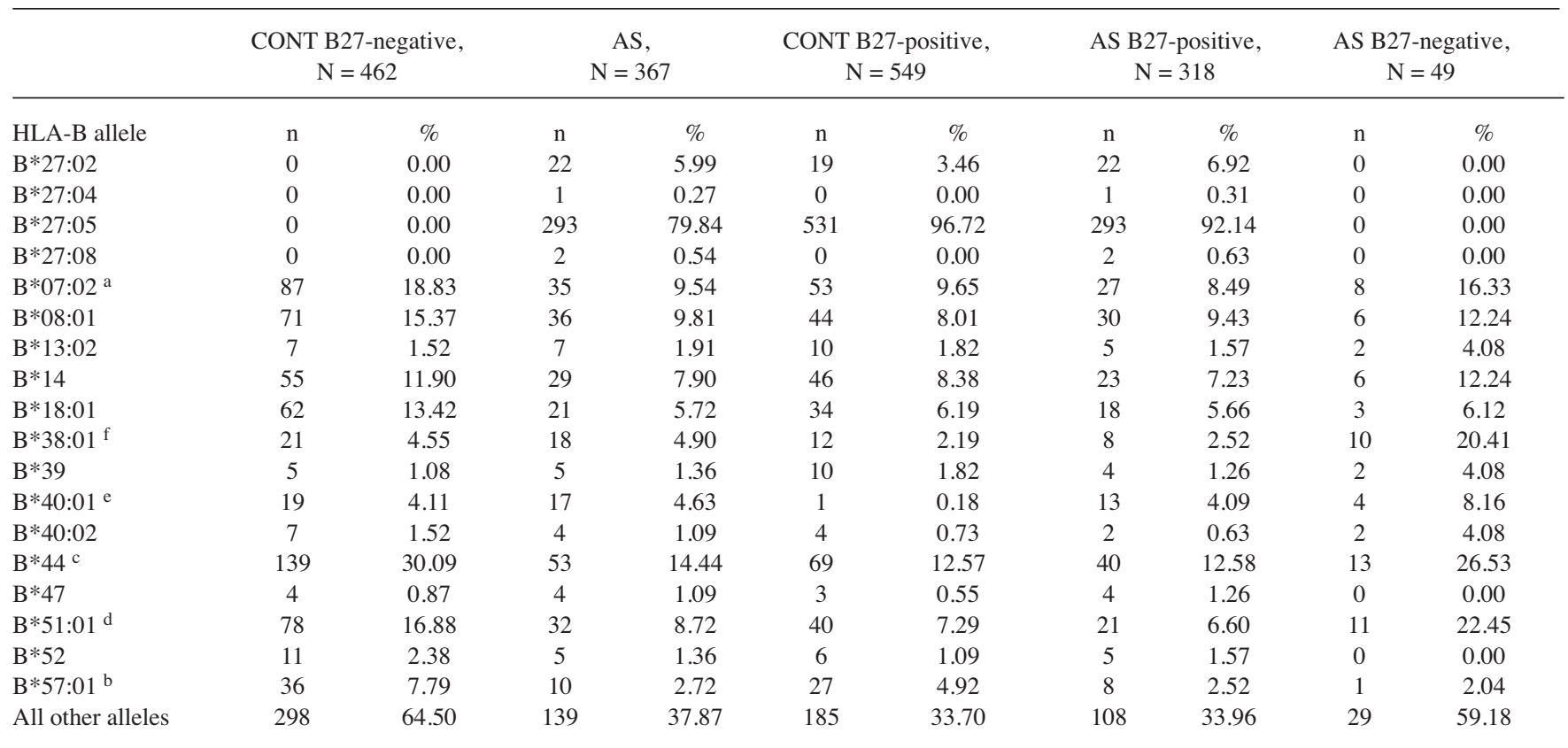

AS versus CONT: ${ }^{\text {a }} 9.5 \%$ vs $18.8 \%, \mathrm{P}_{\mathrm{BONF}}<0.01$, OR $0.45,95 \%$ CI $0.30-0.70 .{ }^{\mathrm{b}} 2.7 \%$ vs $7.8 \%, \mathrm{P}_{\mathrm{c}}<0.005$, OR $0.33,95 \%$ CI $0.16-0.68 .^{\mathrm{c}} 14.4 \%$ vs $30.1 \%$, $\mathrm{P}_{\mathrm{BONF}}<10^{-5}$, OR $0.39,95 \%$ CI $0.28-0.56 .{ }^{\mathrm{d}} 8.7 \%$ vs $16.9 \%, \mathrm{P}_{\mathrm{BONF}}<0.05$, OR 0.47, 95\% CI 0.30-0.73. AS B27-positive vs CONT B27-positive: ${ }^{\text {e }} 4.1 \%$ vs $0.2 \%, \mathrm{P}_{\mathrm{BONF}}<0.001, \mathrm{OR} 23.36,95 \%$ CI 3.04-179.42. AS B27-neg versus CONT: ${ }^{\mathrm{f}} 20.4 \%$ vs $4.6 \%, \mathrm{P}_{\mathrm{BONF}}<0.01$, OR 5.38, $95 \%$ CI $2.37-12.24$. AS: ankylosing spondylitis; $\mathrm{P}_{\mathrm{BONF}}$ : $\mathrm{P}$ value using Bonferroni correction; $\mathrm{P}_{\mathrm{c}}$ : 2-tailed Fisher's exact test; CONT: controls.

Personal non-commercial use only. The Journal of Rheumatology Copyright $\subset$ 2016. All rights reserved. 
Although few samples were analyzed, a strong association of AS with HLA-B*38:01 was seen in HLA-B*27-negative populations. Other studies such as Cortes, et al have not reported this association ${ }^{4}$. It would be interesting to investigate the HLA-B distribution in AS populations in countries where the influence of $\mathrm{B} * 27$ is lower, such as African and Latin American countries ${ }^{10}$. Further research is required to clarify the role of the HLA-B locus in this disease.

ROBERTO DÍAZ-PEÑA, PhD, Department of Immunology, Hospital Universitario Central de Asturias, Oviedo, Spain, and Faculty of Health Sciences, Universidad Autónoma de Chile, Talca, Chile; JOSE RAMÓN VIDAL-CASTIÑEIRA, PhD, Department of Immunology, Hospital Universitario Central de Asturias, Oviedo, Spain; ANTONIO LÓPEZVÁZQUEZ, MD, Department of Immunology, Hospital Universitario Central de Asturias, Oviedo, Spain; CARLOS LÓPEZ-LARREA, PhD, Department of Immunology, Hospital Universitario Central de Asturias, Oviedo, Spain. Address correspondence to C. López-Larrea, Department of Immunology, Hospital Universitario Central de Asturias, C/ Celestino Villamil s/n, 33006-Oviedo, Spain. E-mail: inmuno@hca.es

Supported by Fondecyt grant no. 11130198, and in part by the Spanish grants: Instituto de Salud Carlos III (PI12/02587); Fundación para el Fomento en Asturias de la Investigación Científica Aplicada y la Tecnología (GRUPIN 14-030), and the European Fondos Feder.

\section{REFERENCES}

1. Brown MA, Kennedy LG, MacGregor AJ, Darke C, Duncan E, Shatford JL, et al. Susceptibility to ankylosing spondylitis in twins: the role of genes, HLA, and the environment. Arthritis Rheum 1997;40:1823-8.

2. Robinson PC, Brown MA. Genetics of ankylosing spondylitis. Mol Immunol 2014;57:2-11.

3. Díaz-Peña R, López-Vázquez A, López-Larrea C. Old and new HLA associations with ankylosing spondylitis. Tissue Antigens 2012;80:205-13.
4. Cortes A, Pulit SL, Leo PJ, Pointon JJ, Robinson PC, Weisman MH, et al. Major histocompatibility complex associations of ankylosing spondylitis are complex and involve further epistasis with ERAP1. Nat Commun 2015;6:7146.

5. Díaz-Peña R, Aransay AM, Bruges-Armas J, López-Vázquez A, Rodríguez-Ezpeleta N, Mendibil I, et al. Fine mapping of a major histocompatibility complex in ankylosing spondylitis. Arthritis Rheum 2011;63:3305-12.

6. Díaz-Peña R, Aransay AM, Suárez-Álvarez B, Bruges-Armas J, Rodríguez-Ezpeleta N, Regueiro M, et al. A high density SNP genotyping approach within the $19 \mathrm{q} 13$ chromosome region identifies an association of a CNOT3 polymorphism with ankylosing spondylitis. Ann Rheum Dis 2012;71:714-7.

7. Van de Linden S, Valkenburg HA, Cats A. Evaluation of diagnostic criteria for ankylosing spondylitis. A proposal for modification of the New York criteria. Arthritis Rheum 1984;27:361-8.

8. Evans DM, Spencer CC, Pointon JJ, Su Z, Harvey D, Kochan G, et al. Interaction between ERAP1 and HLA-B27 in ankylosing spondylitis implicates peptide handling in the mechanism for HLA-B27 in disease susceptibility. Nat Genet 2011;43:761-7.

9. Wei JC, Tsai WC, Lin HS, Tsai CY, Chou CT. HLA-B60 and B61 are strongly associated with ankylosing spondylitis in HLA-B27-negative Taiwan Chinese patients. Rheumatology 2004;43:839-42.

10. Benegas M, Muñoz-Gomariz E, Font P, Burgos-Vargas R, Chaves J, Palleiro D, et al. Comparison of the clinical expression of patients with ankylosing spondylitis from Europe and Latin America. J Rheumatol 2012;39:2315-20.

J Rheumatol 2016;43:6; doi:10.3899/jrheum.151096 